\title{
P-56
}

\section{The Naturally Occurring Triterpenes from Pandanus}

\author{
Mohd Sharil Bin Ahmad*, Sadia Sultan, Ummu Amirah Armayni, Nuruljannah Bt Md \\ Yusof, Hannis Fadzillah Mohsin \& Ibtisam Abdul Wahab
}

Faculty of Pharmacy, Universiti Teknologi MARA, 42300 Puncak Alam, Selangor, Malaysia;

E-mail: sharilahmad_3004@yahoo.com

This is a brief introduction of a phytochemical work involving the small screwpines, Pandanus pygmaeus (family: Pandanaceae). The objective is to purify the natural products from the extracts. Previous work discussed other Pandanus, such as $P$. boninensis, $P$. sanderi, and not overlooking the fragrant screwpines, $P$. amaryllifolius. Research papers also published the structures of Pandanus' constituents; particularly the triterpenes. From the literature review, these molecules are the steroidal precursors of the plant hormone and possess biological properties, comparable to the antimicrobial compounds. In an earlier experiment, only one colourless triterpene from the hexane fraction was gained. This compound was later established as stigmasterol, after the crystallization and spectroscopic assessments. Therefore, a quadrupled amount of $P$. pygmaeus was extracted, in order to study other triterpenoids from this species. The adopted methodology consisted of normal silica column chromatography, accompanied with both analytical and preparative thin layer chromatography of the hexane crude extract. The fractionation procedure was planned according to the nonpolarity of each organic component. Nuclear magnetic resonance spectroscopy $\left(500 \mathrm{MHz}, \mathrm{CDCl}_{3}\right)$ was used to identify the molecular structures. The main findings include the isolation of two possible triterpenes. Blue spots were visualised when vanillin was utilised as the spray reagent. Preliminary spectroscopic evidences suggested that the carbon arrangement of the molecules belong to other than the stigmasterol. On the other hand, the presence of an olefinic system might include a vinyl proton, directly attached to the carbon-carbon double bond. Nevertheless, this fragment is unlike the one from the antitubercular triterpene of $P$. sanderi, where an exomethylene moiety was instead characterised. The purification of these triterpenes should be highlighted, since this is the second report of such occurrence from $P$. pygmaeus. Ongoing work also incorporates the antibacterial and antifungal evaluation of these compounds.

Keywords: Pandanus, triterpenes. 